\title{
Global-Soziale Marktwirtschaft und die Flüchtlingsfrage ${ }^{1}$
}

Carl Christian von Weizsäcker

Senior Research Fellow am Max Planck Institute for Research on Collective Goods

Kurt-Schumacher-Str. 10, 53133 Bonn

\section{Received July 18 2016; Accepted November 92016}

Zusammenfassung: Auslöser meiner Überlegungen ist die akute Flüchtlingskrise. Es spricht manches dafür, dass sich diese Krise
nicht einfach mit einigen administrativen Maßnahmen der Zuwanderungsbeschränkung in kurzer Frist in Luft
auflösen wird. Vermutlich ist sie Symptom für eine neue Welle der weltweiten Angleichung von Lebensbedin-
gungen, die sich unter anderem in großen Wanderungen niederschlägt. Es ist dann im Interesse des Nordens
diesen Angleichungsprozess zu beschleunigen, um so den Anreiz des Wanderns aus dem Süden in den Nor-
den zu dämpfen. Eine zentrale Komponente des nördlichen Erfolgsmodells, die Soziale Marktwirtschaft,
sollte daher möglichst rasch globalisiert werden. Diesem Ziel einer möglichst zügigen Verwirklichung einer
Global-Sozialen Marktwirtschaft dient am besten ein Kulturwandel im Süden in Richtung auf das das nördli-
che Erfolgsmodell. Dieser Kulturwandel kann vor allem angestoßen werden durch Exporterfolge des Südens
in den Norden. Hierzu eignet sich eine Außenwirtschaftspolitik des Nordens, die einen Exportüberschuss
des Südens hervorbringt und auf diese Weise im Süden möglichst viele produktive Arbeitsplätze schafft.
Der Norden würde von einer solchen Politik nicht nur deshalb gewinnen, weil auf diese Weise der Süd-Nord-
Wanderungsdruck gemildert wird. Darüber hinaus kann der Norden auch durch eine verbesserte internatio-
nale Arbeitsteilung gewinnen. Allerdings ist es für den Erfolg einer solchen Politik auch erforderlich, dass sich
der Norden auf die damit verbundenen institutionellen Veränderungen einlässt. Insbesondere muss akzeptiert
werden, dass sich die komparativen Vorteile in der Güterproduktion verschieben und dass auch Institutionen wie
zum Beispiel die staatliche Schuldenbremse modifiziert, grundlegend geändert oder ganz abgeschafft werden.
Internationale Migration • Wirtschaftswachstum offener Volkswirtschaften • Zinsen
C De Gruyter Open Sp. z o.0.

\section{Einleitung}

„Arbeitsplätze für die Dritte Welt" sollte die Devise sein, die auf die Flüchtlingskrise antwortet. Die aktuelle Diskussion klebt jedoch am Status Quo in Deutschland. Ihn zu erhalten scheint das Hauptanliegen zu sein. Das Denken verläuft in eingefahrenen Gleisen. Alternativlösungen, die erheblich vom Status Quo abweichen, kommen gar nicht in den Tunnelblick der Eliten

1 Schriftliche Fassung der Wilhelm Röpke Vorlesung, gehalten in Erfurt am 11. Februar 2016. und Wähler. Die Politische Ökonomie kann diesem Missstand abhelfen, indem sie die Handlungsmöglichkeiten aufweist, die bei einer Horizonterweiterung ins Auge fallen.

„Jenseits von Angebot und Nachfrage“ ist ein Spätwerk von Wilhelm Röpke (Röpke 1958). Auch er versuchte darin eine Horizonterweiterung. Bemerkenswert zum Beispiel Röpkes Prophezeiung aus dem Jahr 1958, dass es mit dem zentral-planwirtschaftlichen Wirtschaftssystem ein Ende haben werde, weil es notwendigerweise auf einer Unterdrückung der persönlichen Freiheit fußt. Hier nun 
erlaube ich mir eine Horizonterweiterung "Jenseits von Schuldenbremse und gesetzlichem Mindestlohn“.

Nicht fern von Erfurt entstanden im Jahr 1801 die folgenden Verse:

„Nichts Bessers weiß ich mir an Sonn- und Feiertagen

Als ein Gespräch von Krieg und Kriegsgeschrei.

Wenn hinten weit in der Türkei

Die Völker aufeinanderschlagen.

Man steht am Fenster, trinkt sein Gläschen aus

Und sieht den Fluss hinab die bunten Schiffe gleiten;

Dann kehrt man abends froh nach Haus,

Und segnet Fried und Friedenszeiten."

„Herr Nachbar, ja! So lass ichs auch geschehn

Sie mögen sich die Köpfe spalten,

Mag alles durcheinander gehn;

Doch nur zuhause bleibs beim alten." (Goethe 1808).

Goethe ironisiert hier, kurz bevor Faust seinen Mephisto trifft, den Tunnelblick seiner Mitbürger am Vorabend der großen Umwälzung, der Französischen Revolution, die das Tor zur Moderne öffnete. Und diese Moderne wurde bei allen Turbulenzen, Verbrechen und Desastern letztlich zu einem Erfolg des materiellen Lebens, den man sich zuvor gar nicht hat vorstellen können.

Vielleicht stehen wir heute vor einer neuen Welle in der Entwicklung dieser Moderne. Hierzu im Folgenden einige Gedanken.

\section{Der Drang zu immer mehr Gleichheit}

Von Alexis de Tocqueville stammt die Diagnose eines unausweichlichen welthistorischen Drangs zu immer mehr Gleichheit unter den Menschen (De Tocqueville 1835/1840). Und vieles, was wir seither in der Weltgeschichte beobachten konnten, entspricht dieser Diagnose. Die Sklaverei wurde in den abendländisch geprägten Teilen der Welt abgeschafft. Auf das Kolonialzeitalter folgte die Selbständigkeit der Kolonien. Das 20. Jahrhundert sah die universelle Einführung des Frauenwahlrechts. In den reichen Ländern wurde ein umfassender Sozialstaat aufgebaut. Der Rassismus wurde als Weltanschauung geächtet; und es wurden rassistische Praktiken massiv zurückgedrängt. In der zweiten Hälfte des 20. Jahrhunderts haben sich die materiellen Lebensbedingungen, global gesehen, angeglichen. Hierzu nur drei Fakten: Während sich in den letzten 50 Jahren die Lebenserwartung der Gesamtbevölkerung in
Deutschland und in anderen reichen Ländern um rund 10 Jahre erhöht hat, ist die Lebenserwartung der Weltbevölkerung als ganzer um mindestens 17 Jahre gestiegen. Die Ungleichheit der Einkommen im Weltmaßstab hat abgenommen, nicht zuletzt wegen des massiven Zuwachses der Einkommen in großen Schwellenländern wie China und Indien. Und für die Weltbevölkerung können wir in den allerletzten Jahrzehnten konstatieren: Der Weltbevölkerungs-Anteil der Menschen unter der absoluten Armutsgrenze hat sich zwischen den Jahren 1990 und 2012, also innerhalb von nur 22 Jahren, auf ein Drittel reduziert. Die Anzahl der Menschen unterhalb der absoluten Armutsgrenze hat sich von 1990 bis 2012 halbiert (Weltbank 2016).

Ich erlaube mir, Sie in diesem Vortrag mit der Hypothese zu konfrontieren, dass der uns alle in Deutschland heute bewegende Flüchtlingsstrom Teil einer neuen Welle zu mehr Gleichheit unter der Weltbevölkerung ist. Unter dieser Hypothese leite ich dann bestimmte Vorschläge $a b$, wie darauf zu reagieren wäre.

Man spricht vom Tocqueville-Paradoxon: Schon Tocqueville (1856) hat anhand der Vorgeschichte und der Geschichte der Französischen Revolution konstatiert: Es war gerade der zunehmende fortschrittliche Reformgeist im Zeitalter Ludwigs XVI, der mit dem an Verbesserungen schon Erreichten nicht zufrieden war und der schließlich in der Revolution mündete. Mehr Gleichheit generiert eine steigende Unzufriedenheit mit der noch vorhandenen Ungleichheit und drängt daher auf immer noch mehr Gleichheit.

Und so beobachten wir auch heute im öffentlichen Diskurs, in den Medien und in der Politik die Betonung der Ungleichheit und einer ansteigenden Ungleichheit bei Einkommen und Vermögen. Ich halte diese Beobachtung gestiegener Ungleichheit für eine Folge eines Tunnelblicks, der sich nur auf einen ganz kleinen Ausschnitt von Indikatoren konzentriert. Es ist richtig, dass in den meisten reichen Staaten das verfügbare Arbeitseinkommen heute ungleicher verteilt ist als früher. Es ist richtig, dass die Lohnquote als Anteil am Volkseinkommen im Verlauf der Zeit abgenommen hat. Auch in der Vermögensverteilung, so wie sie herkömmlich gemessen wird, sieht man gestiegene Ungleichheit. Indessen ist ein sinnvoller Begriff des Lebensstandards weiter zu fassen als nur mittels der beiden Begriffe Einkommen und Geld- und Sachvermögen.

Betrachten wir die Ungleichheit auf nationaler Ebene: Erstens, ein wichtiger Teil der Ungleichheit bei den Lohneinkommen ist die Ungleichheit in den Löhnen zwischen Männern und Frauen. Aber in dieser Ungleichheit scheint gar nicht auf, dass es herkömmlich 
viele Frauen gab, die gar keiner Berufsarbeit nachgingen. Sie wurden bei der Abschätzung der Ungleichheit der Lohneinkommen gar nicht berücksichtigt. Würde man bei den Personen im Alter zwischen 20 und 65 Jahren die nicht Berufstätigen mit einem Arbeitseinkommen von Null mitberücksichtigen, dann wäre vermutlich die deutsche Lohnungleichheit im Jahr 1965 höher gewesen als im Jahr 2015. Zweitens, analoge Überlegungen lassen sich bei den Einwanderern aus Niedriglohnländern anstellen: Ein bedeutsamer Teil der Lohnungleichheit in Deutschland besteht darin, dass diese Einwanderer bzw. ihre Kinder im Durchschnitt niedrigere Löhne erhalten als die Lohnbezieher, die und deren Eltern in Deutschland geboren sind. Aber die Einwanderer-Kinder und ihre Eltern wären wesentlich schlechter gestellt, wenn sie nicht eingewandert wären. Das Faktum dieser Einwanderung hat sie materiell wesentlich bessergestellt; aber sie tragen zur gemessenen Ungleichheit der Löhne bei. Drittens, gleiches gilt in Deutschland für den Wiedervereinigungseffekt. Die Löhne sind in den neuen Bundesländern nach wie vor geringer als in Westdeutschland. Insofern hat die Wiedervereinigung zu höherer Lohnungleichheit beigetragen, so wie sie gemessen wird. Jedoch war für die ganz große Mehrheit der Ostdeutschen die Wiedervereinigung ein großer materieller Zugewinn. Hätte man vor der Wiedervereinigung eine Lohnverteilung für Gesamtdeutschland aufgestellt, so wäre die Lohnungleichheit noch höher ausgefallen als nach der Wiedervereinigung und als heute. Viertens, bei den monetären Netto-Einkommen der Menschen sind die gesellschaftlichen Ausgaben für das Gesundheitssystem weitgehend ausgespart, weil diese zu einem erheblichen Teil über öffentliche Kassen finanziert werden. Die Ungleichheit in der Nutzung dieser Gesundheitsausgaben ist jedoch weitaus geringer als bei den Einkommen oder den Vermögen. Für die große Mehrheit der Bevölkerung sind die durch staatliche Abgaben finanzierten Gesundheitsleistungen unabhängig vom persönlichen Einkommen. Diese Gesundheitsausgaben haben in den letzten Jahrzehnten einen wachsenden Anteil am Sozialprodukt ausgewiesen und damit zu einer gleichmäßigeren Verteilung in der Nutznießung knapper Ressourcen beigetragen. Fünftens, analoge Überlegungen resultieren aus der gestiegenen Lebenserwartung. Während die durchschnittliche Rentenbezugsdauer oder Pensionsbezugsdauer vor einem halben Jahrhundert bei rund 10 Jahren lag, liegt sie heute bei 20 Jahren. Das durchschnittliche Arbeitsleben hat sich jedoch - selbst unter Einbeziehung der berufsvorbereitenden Ausbildung - nicht verlängert. Daher ist das Vermögen in der Form von Renten- und Pensionsanwartschaften relativ zum aktuellen Arbeitseinkommen stark gestiegen. Würde man diese Anwartschaften gemäß den Kalkulationsprinzipien der privaten Lebensversicherung dem privaten Vermögen zuschlagen, sähe nicht nur die Vermögensverteilung wesentlich gleichmäßiger aus; vor allem hätte es angesichts der gestiegenen Anwartschaftswerte einen wesentlichen Beitrag zu einer Vergleichsmäßigung der Vermögensverteilung im Zeitverlauf gegeben, der in der offiziellen Vermögensverteilungs-Statistik nicht berücksichtigt wird. Sechstens, der Zugang zur höheren Bildung an Universitäten und Fachhochschulen ist seit Mitte des 20. Jahrhunderts in fast allen reichen Ländern massiv demokratisiert worden. Etwa die Hälfte eines Altersjahrgangs besucht unter den heute jungen Leuten die Hochschule. Heute studieren in fast allen reichen Ländern mehr Frauen als Männer an den Hochschulen. Die Bildungschancen und damit die Berufseinstiegschancen sind heute weit gleichmäßiger verteilt als vor einem halben Jahrhundert. Die Chancengleichheit ist massiv gestiegen. Siebtens, schließlich sei auf den egalisierenden Effekt gestiegener Preisstabilität verwiesen. Während reichen Menschen die Zukunftsvorsorge auch in Zeiten mit instabiler Währung und schlecht voraussehbarer Inflation gelingt, gilt dies nicht für den kleinen Sparer. Er ist bei seiner Zukunftsvorsorge neben den sozialstaatlichen Einrichtungen auch darauf angewiesen, dass seine Ersparnisse real erhalten werden. Das wird wesentlich erleichtert, wenn Preisstabilität erwartet werden kann. Nun gibt es keinen Zweifel, dass heute die Preisstabilität besser gelingt als dies zuvor, insbesondere in der ersten Hälfte des 20. Jahrhunderts, der Fall war.

Ich bin mir ziemlich sicher, dass selbst auf nationaler Ebene in Deutschland und in vergleichbaren Staaten die Gleichheit in den materiellen Lebensbedingungen zugenommen hat, wenn man die genannten Effekte (und weitere) mitberücksichtigen würde.

Aber entscheidend ist vor allem, dass die weltweite Verteilung von materiellen Lebenschancen gleichmäßiger geworden ist.

Aber nichtsdestoweniger gibt es das TocquevilleParadoxon. Die vermehrte Gleichheit führt auch im Weltmaßstab zu einem vermehrten Drängen nach noch mehr Gleichheit. Die Globalisierung, die wir in den vergangenen Jahrzehnten erlebt haben, bringt es mit sich, dass die Stoßrichtung vermehrter Gleichheit heute darauf zielt, die Unterschiede zwischen dem Norden - den reichen Ländern - und dem Süden zu vermindern. Es geht um die Beschleunigung des Aufholprozesses des Südens, der ja ohnehin, wie oben schon diskutiert, im Gange ist. 


\section{Das Erfolgsmodell der reichen Länder und die Wanderung von Arm zu Reich}

Zuerst die Erklärung, weshalb es diesen großen Abstand zwischen Norden und Süden gibt. Wie unser neuester Nobelpreisträger, Angus Deaton, in seinem Buch "The Great Escape" dargestellt hat (Deaton 2013), gelang es dem Norden, seine Bevölkerung aus dem mehrheitlichen Zustand am Existenzminimum heraus zu führen, indem Institutionen geschaffen wurden, die der Wohlstandssteigerung förderlich waren. Es war dies kein Generalplan, sondern ein welthistorisches Experiment von Versuch und Irrtum. Kurz zusammengefasst bestehen die Institutionen, die diese "Große Transformation“ (Polanyi 1943) schufen, aus folgenden Elementen: Ein Rechtsstaat, der Rechtssicherheit und die Gleichheit vor dem Gesetz gewährt; das Gewaltmonopol des Staates, das Bürgerkrieg verhindert; die Gewaltenteilung, die Demokratie und individuelle Freiheit ermöglicht; eine wettbewerblich verfasste Marktwirtschaft, die in diesem staatlichen Rahmen Anreize für Effizienz und materiellen Fortschritt schafft; Freiheit der Wissenschaft, die neues, vielfach nützliches Wissen generiert; Meinungsfreiheit und Mehrheitsprinzip, die für gewaltfreie Formen des Machtwechsels und somit für gesellschaftliche Integration der meisten Bürger sorgen; ein Sozialstaat, der bei allen Bürgern ein Interesse an der Stabilität der öffentlichen Zustände generiert.

Die aktuelle Flüchtlingswelle reiht sich ein in eine große Anzahl von Wanderungsbewegungen, die wir auch als Völkerwanderungen bezeichnen können. Völkerwanderungen haben ein einfaches Grundmuster. Die Menschen fliehen aus der Heimat, wo Not, Krieg und Übervölkerung herrschen, und streben dorthin, wo Brot, Sicherheit und Unterkommen die Regel sind. Die konkrete Veranlassung von plötzlichen Spurts in Wanderungsbewegungen ist allerdings schwer voraussagbar. Es hilft hier, auf den Hayekschen Begriff der Mustervoraussagen zurückzugreifen (Hayek 1972). Man kann die von den Menschen subjektiv empfundenen KostenNutzen-Verhältnisse einer Auswanderung und Einwanderung begrifflich erfassen; darauf kann man eine Mustervoraussage aufbauen. Aber der Betrachter hat ex ante nicht das Wissen über den Informationsstand potentieller Wanderer. Daher hilft inm diese Mustervoraussage nur beschränkt oder gar nicht, Zeitpunkt und Ausmaß einer konkreten Wanderungswelle vorherzusagen.

Im Sinne einer Mustervoraussage kann man jedoch folgendes konstatieren: Je größer das Wohlstandgefälle zwischen zwei Regionen ist, desto wahrscheinlicher ist ceteris paribus die Tendenz der Auswanderung aus der einen und der Einwanderung in die andere Region.

In der gegenwärtigen Debatte, wie einleitend schon festgestellt, konzentriert man sich in den reichen Ländern auf das Thema der Abwehr der Zuwanderung. Hierzu will ich in diesem Vortrag nicht viel sagen. Vielmehr beschränke ich mich auf die andere Antwort auf die Frage nach einer Begrenzung der Zuwanderung: Die Verminderung des Anreizes, aus den Ländern des Südens auszuwandern. Diese Antwort wird in der gegenwärtigen Rhetorik zwar immer mitgeführt; aber sie wird verkürzt auf den außenpolitisch-militärpolitischen Aspekt der Beendigung von Bürgerkriegen, sei es in Syrien, sei es im Irak, sei es in Afghanistan, sei es in Somalia, sei es in Westafrika. Die Frage nach den ökonomisch-gesellschaftlichen Ursachen dieser Bürgerkriege gerät völlig in den Hintergrund. Auf diese werfe ich mein Augenmerk.

Ich tue dies, wissend, dass man damit nur eine langfristig wirksame Antwort finden kann. Indessen, wir sollten nicht Opfer der „Vordringlichkeit des Befristeten“ (Luhmann 1968) werden. Kurieren an den Symptomen wird angesichts der Wucht des zu lösenden Problems nicht ausreichen.

\section{Globalisierung des sozialen Ausgleichs zwecks Eindämmung von Wanderungen}

Mir geht es damit um die Antwort auf die Frage: Was können wir tun, um das Wohlstandsgefälle zwischen dem Norden und dem Süden zu mindern? Und dies unter der Nebenbedingung, dass der Wohlstand des Nordens darunter nicht leidet. Damit bewegen wir uns auf die Frage zu: Was können wir im Norden tun, um den Wohlstand im Süden zu steigern?

In dem Versuch, hier eine Antwort zu finden, greife ich zurück auf den Begriff der Sozialen Marktwirtschaft. Die Marktwirtschaft ist trotz aller Effizienzvorteile doch nur mehrheitsfähig, wenn sie verbunden ist mit einem Sozialstaat, der für einen gewissen Ausgleich zwischen erfolgreichen und weniger erfolgreichen Teilnehmern am großen Wirtschafts-Spiel sorgt. Schon die Kathedersozialisten des 19. Jahrhunderts verstanden ihre Vorstellungen von Sozialpolitik (Socialpolitik) als einen Beitrag zur politischen Stabilisierung der bürgerlichen Gesellschaft. Der erfolgreiche Beginn der von den Kathedersozialisten erdachten und von Bismarck begonnenen modernen Sozialpolitik erlaubte es dem Gesetzgeber, das Sozialistenverbot aufzuheben und damit ganz wesentlich zum sozialen Frieden beizutragen. Die Weiterentwicklung des Sozialstaates nach dem Zweiten Weltkrieg mag bei 
allen Übertreibungen, die natüllich vorkamen, auch die Legitimität des Marktes in Deutschland, aber vor allem auch in Europa gestärkt haben. Dass die europäische Integration den Weg über die Schaffung eines Gemeinsamen Marktes genommen hat, war politisch wohl nur möglich, weil in allen Mitgliedstaaten sozialstaatliche Institutionen vorhanden waren, mit deren Hilfe die Abfederung von Neuverteilungen gelang, die die unausweichliche Folge des Wettbewerbs im Gemeinsamen Markt waren. Bei allen Fehlentwicklungen in den verschiedenen Sozialsystemen der Mitgliedsstaaten muss man diese Legitimierungsfunktion des Sozialstaates für die Marktwirtschaft anerkennen.

Aber das oben skizzierte Institutionensystem der bürgerlichen Gesellschaft war so erfolgreich, dass gerade deshalb neue Probleme auftauchten. "The Death of Distance" ist der Titel eines Buches von Frances Cairncross (1997). Dieser Titel bringt eine der Errungenschaften der Moderne auf den Punkt. Die Kosten der Distanzüberwindung sind für Informationen praktisch null geworden. Sie sind für Güter- und Personentransporte massiv gefallen - und dieser Trend hält an. Damit entsteht ein enormes Globalisierungspotential, das zu einem großen Teil auch genutzt wird. In unserem Zusammenhang geht es um die Abnahme der Wanderungskosten zwischen Regionen unterschiedlichen Wohlstands. Bei gleichem Wohlstandsgefälle führt diese Abnahme zu steigenden Wanderungszahlen. Oder anders ausgedrückt: Sinken die Wanderungskosten, so kann ein Zuwachs bei den Wanderungen nur verhindert werden, wenn das Wohlstandsgefälle zwischen Nord und Süd kleiner wird.

Man kann auf sinkende natürliche Wanderungskosten auch dadurch reagieren, dass man versucht, die Wanderungskosten künstlich zu erhöhen. Ein Stück weit wird dies in der kurzen Frist unvermeidlich sein; aber ich glaube nicht, dass dies auf Dauer die einzige Antwort sein kann - sofern man die humanitären Werte des Nordens nicht über Bord werfen will. Jedenfalls diskutiere ich in diesem Vortrag nicht die künstliche Erhöhung der Wanderungskosten.

Damit gehe ich hier dem Gedanken nach: Wie kann angesichts sinkender Wanderungskosten der Wohlstand im Süden so schnell gesteigert werden, dass die Wanderung in den Norden diesen nicht destabilisiert?

\section{Das Beispiel China}

Hierzu lohnt sich ein Blick in die jüngere Geschichte und auch in die Dogmengeschichte. Es gibt ja ein Phänomen unglaublichen wirtschaftlichen Aufstiegs: China. Im Jahr 1980 lebten drei Viertel aller Einwohner der Volksrepublik China unter der absoluten Armutsgrenze.
Diese wird allgemein festgemacht bei einem Einkommen von einem US-Dollar der Kaufkraft des Jahres 1993 pro Kopf und Tag. Heute gibt es unter den HanChinesen praktisch niemand mehr, der mit einem Dollar pro Tag auskommen muss. Es mag unter bestimmten ethnischen Minderheiten (Tibeter, Uiguren etc.) noch Menschen unter dieser absoluten Armutsgrenze geben (Weltbank 2016). Ein Prozess des wirtschaftlichen Wachstums hat stattgefunden, der seinesgleichen in der Wirtschaftsgeschichte sucht.

Ich interpretiere diesen chinesischen Erfolg als Begleiterscheinung eines fundamentalen Kulturwandels. China hat gelernt, wie Marktwirtschaft funktioniert. Die Lehrmeister Chinas waren die Länder des Nordens. Das Lehrbuch war der chinesische Export in jene Länder (generell zu China: Kissinger 2011).

Ich verweise in diesem Zusammenhang gern auf das Buch „Violence and Social Orders" von North, Weingast und Wallis. Die Autoren definieren zwei Zustände, in denen sich Gemeinwesen befinden können, die sie Natural State und Open Access Order nennen. Die vormodernen Gemeinwesen sind Natural States; die heute reichen Staaten entsprechen der Open Access Order. Einer der wesentlichen Unterschiede zwischen beiden Zuständen ist die Art und Weise, wie mit dem Problem der Anwendung physischer Gewalt umgegangen wird. Im modernen Staat (Open Access Order) gibt es das Gewaltmonopol des Staates. Dieses fehlt im Natural State. In diesem müssen andere Wege gefunden werden, wie die Ausübung von Gewalt minimiert wird. Die soziale Struktur des Natural State kann sehr weitgehend interpretiert werden als eine Antwort auf die Frage, wie man ohne staatliches Gewaltmonopol Gewalt und Bürgerkrieg verhindern kann. Entscheidend für unseren Zusammenhang ist, dass sich im Natural State soziale Beziehungen herausbilden müssen, die den einzelnen Menschen einen Status zuschreiben, welcher ihre Handlungsmöglichkeiten stark begrenzt. Damit kann aber auch die Arbeitsteilung als Hauptquelle hoher Produktivität nur sehr beschränkt verwirklicht werden. Die sozialen Beziehungen in den traditionalen Gesellschaften sind sehr stark auf Verwandtschaft gegründet. Aus innen ergeben sich Handlungsweisen, die man aus Sicht des modernen Staates als Korruption bezeichnen würde. Sie sind aber im Rahmen der traditionalen Gesellschaft insofern im Interesse des Gemeinwohls, als sie die Bedingung dafür sind, dass die Anwendung von physischer Gewalt zurückgedrängt werden kann. In den traditionalen Gesellschaften gibt es auch nicht die Trennung von Macht und Reichtum. Denn es gehört dort zu den Gewaltvermeidungs-Methoden, dass Besitz und Eigentum ständig umverteilt wird: Von denen, deren Macht schwindet, zu denen, deren Macht zunimmt. 
Demgegenüber ist es ein Charakteristikum des modernen Rechtsstaats, dass Macht und Reichtum auseinanderfallen können und überwiegend auch tatsächlich auseinanderfallen. North et al. interessiert in diesem Zusammenhang die Frage, wie sich die Open Access Order aus dem Natural State entwickeln kann und in der Geschichte entwickelt hat. Es ist dies eine faszinierende Sichtweise auf die Sozialgeschichte der europäischen Neuzeit.

Natürlich gibt es große Unterschiede zwischen den Ländern. Man kann nicht einfach von einem Land auf das andere schließen. Dennoch ist China auch ein Lehrbeispiel für andere Staaten. China durchlief verschiedene, sehr unterschiedliche Phasen der Modernisierung. Die vom Westen erzwungene Öffnung der Grenzen im Verlauf des 19. Jahrhunderts, mitsamt dem Opiumkrieg unter dem Segel des freien Warenaustauschs, endete schließlich in der Abschaffung der Monarchie. Unter Sun Yat-sen und dann Tschiang Kai-Shek entstand ein reformwilliges Regime, das aber, nicht zuletzt veranlasst durch die japanische Invasion, zur Militärdiktatur mutierte. Diese brach indessen unter den großen Spannungen zwischen Arm und Reich, zwischen Stadt und Land zusammen. Es folgte die Modernisierung mit kollektivistischem Vorzeichen unter der Führung der KP Chinas mit Mao Tse-dung an der Spitze. Diese kulminierte in der Kulturrevolution der sechziger und siebziger Jahre mit ihren katastrophalen Folgen für den Lebensstandard. Die Kehrtwende zur Marktwirtschaft, eingeleitet unter der Führung von Deng Xiaoping, führte zu dem oben schon erwähnten Wachstumsschub. Aber diese Kehre zur Marktwirtschaft konnte schon profitieren von Maßnahmen der Mao-Zeit, so insbesondere von der Ein-Kind-Politik und einer erheblichen Verbesserung des Gesundheitswesens. Daher war die Demographie Chinas schon zu Beginn dieser Ära die eines fortgeschrittenen Industrielandes, ohne schon ein solches zu sein.

Die chinesische Kultur blieb über all diese Zeit stark geprägt vom Familienzusammenhalt. Die Sorge der Eltern in China für eine gute Schulausbildung der Kinder ist sprichwörtlich. Während dieser Familiensinn jedoch in der Zeit vor der Wende zur modernen Marktwirtschaft zu Zuständen führte, die man im Westen Protektion, Vetternwirtschaft oder Korruption nennt, ist dies heute anders. Zwar gibt es diese Erscheinungen immer noch zur Genüge, aber daneben erhielt man die Möglichkeit, im Weltmarkt erfolgreich zu sein. Daher verlagerte sich das Verhalten auf Aktivitäten, die nunmehr auch volkswirtschaftlich von großem Nutzen waren. Denn im internationalen Wettbewerb ist das ausschlaggebende Kriterium des Erfolgs, dass man Ware mit akzeptabler Qualität zu günstigen Preisen pünktlich liefern kann. Die
Spielregeln der Marktwirtschaft wurden anhand dieses Exports gelernt. Hierbei war sicher auch hilfreich, dass taiwanesische Unternehmer die Chance erhielten, auch in der Volksrepublik China tätig zu werden und so ihre Erfahrungen aus dem internationalen Geschäft zum gegenseitigen Vorteil einzubringen. Auch Honkong, das bis zum Jahr 1997 britische Kronkolonie war und danach als Teil der Volksrepublik einen politischen Sonderstatus behielt, diente als Drehscheibe für das chinesische Exportgeschäft.

Aber selbst wenn aus den eben aufgeführten Gründen die chinesische Erfahrung nicht eins zu eins auf andere Länder übertragen werden kann, bleibt der Kern der Aussage richtig: Es ging um das Nachholen der Erfolge der Open Access Society - und hierfür waren diese selbst die besten Lehrmeister, indem man ihnen Waren verkaufen konnte. Es ist daher kein Zufall, dass dieser rasche Wachstumsprozess von einem ständigen, quasi strukturellen Exportüberschuss begleitet war.

Gegen diesen Exporterfolg Chinas regte sich immer wieder Widerstand in den Ländern des Nordens. Der Renminbi galt als unterbewertet. Indessen sollte nicht vergessen werden, dass diesem Kapitalexport seitens eines Entwicklungslandes auch direkte ökonomische Vorteile für den Norden gegenüberstanden. Die chinesische Teilhabe am Weltmarkt hatte in den reichen Ländern nicht nur den Verlust von bestimmten Arbeitsplätzen, etwa in der Bekleidungsindustrie oder anderen Konsumgüterbranchen, zur Folge. Zugleich wurden Arbeitsplätze in den Branchen geschaffen, die von Exporten nach China profitierten. Ferner bedeutete die von China ausgehende Sparschwemme, dass die Zentralbanken einen weitaus expansiveren Kurs fahren konnten, ohne Inflation herauf zu beschwören. Dieser Kurs hat ohne Zweifel eine große Anzahl von Arbeitsplätzen geschaffen.

\section{Die heutige Bedeutung von Friedrich List: Arbeitsplätze für den Süden durch Globalisierung des nördlichen Erfolgsmodells}

In seinem „Nationalen System der politischen Ökonomie" schrieb Friedrich List (1841) gegen die Freihandelslehre der Klassiker an. $\mathrm{Er}$ entwickelt eine Dreistufenlehre der wirtschaftlichen Entwicklung. In den Agrarstaaten der untersten oder ersten Stufe sei der Freihandel angemessen; ebenso in der dritten Stufe der vollen industriellen Entwicklung. Er identifiziert das Großbritannien seiner Zeit mit dieser dritten Stufe. In der zweiten Stufe befand sich damals das noch politisch 
zersplitterte Deutschland. Hier empfiehlt List Schutzzölle für die noch in den Kinderschuhen steckende Industrie. Später hat man in der neoklassischen Außenhandelstheorie Lists Infant-Industry-Argument mit positiven Externalitäten begründet, die sich in diesem Industrialisierungsprozess ergeben. Es geht bei List jedoch um mehr; es geht im Grunde um eine jeweils fundamentale Gesellschaftsveränderung, um die Transformation einer traditionalen Gesellschaft in eine moderne Gesellschaft, um ein Nachvollziehen der Großen Transformation.

Meine Position in dieser Arbeit ist im Grunde eine lineare Fortsetzung der Listschen Theorie. Einzig der Blickwinkel ist ein anderer. Während List aus der Sicht des (damaligen) Südens argumentierte, plädiere ich, fast zwei Jahrhunderte später aus der Sicht des Nordens, der, aus den oben diskutierten Gründen zunehmender Wanderungen, heute daran interessiert sein muss, den Entwicklungsprozess des Südens zu beschleunigen. Damals, zu Lists Zeiten, sollte die inländische Industrialisierung den Kulturwandel im Süden hervorbringen. Heute soll das Lernen vom Kunden aus dem Norden hinzukommen. Damals ging es um das Nachvollziehen eines Kulturwandels innerhalb der abendländischen Zivilisation. Heute geht es um die Übernahme des modernen abendländischen Lebensstils durch die ganze Welt.

Die zentrale Idee kann auch so ausgedrückt werden: Den Kulturwandel vom Kunden lernen. Vom Kunden lernen ist aber ohnehin eine wohlbekannte Erkenntnis der Innovationsforschung. Es sind gerade die Kunden, die dem Unternehmer den Hinweis geben, wie das Design seines neuen Produktes inn zum Erfolg führt.

Die Politikempfehlung für den Norden ist damit, dass man den Ländern des Südens ermöglicht, möglichst viel an Waren und Dienstleistungen an den Norden zu liefern, um damit den Aufholprozess zum Norden zu beschleunigen und so eine Dämpfung des Wanderungsdrangs in den Norden zu erreichen.

Damit wird im Süden Wachstum generiert. Und vor allem: Es werden Arbeitsplätze geschaffen, sodass es sich lohnt, dort zu bleiben.

\section{Abbau von Handelshemmnissen bei Importen aus dem Süden}

Geht eine derartige Politik des Nordens aber nicht zulasten des Lebensstandards im Norden? Gehen damit nicht viele Arbeitsplätze im Norden verloren? Die Antwort ist: Es gehen Arbeitsplätze verloren; aber andere, und wahrscheinlich genauso viele oder mehr entstehen, sofern man es richtig anstellt. Im Folgenden werde ich diese Antwort begründen.
Es gibt für den Norden im Wesentlichen zwei Verfahren, die dazu beitragen können, die Warenexporte des Südens in den Norden zu stimulieren, um so das Wachstum im Süden zu beschleunigen. Das eine Verfahren wird von den meisten Ökonomen befürwortet: Abbau von Handelshemmnissen für Importe aus dem Süden. Ich werde hierzu in diesem Vortrag wenig sagen.

Nur so viel als Merkposten. Wenn es um Importe aus Niedriglohnländern geht, ist das in der Öffentlichkeit der reichen Länder dominierende Denken beherrscht von der Angst eines Verlusts von Arbeitsplätzen. Die politische Durchsetzbarkeit einer weiteren Liberalisierung von Importen aus dem Süden ist daher ein großes Problem. Sie ist wohl nur dann gegeben, wenn die Wählerschaft darauf vertraut, dass die Politik das heimische Arbeitsplatzproblem mittels kompensierender Maßnahmen im Griff hat. Die folgenden Überlegungen dienen insbesondere dazu, plausibel zu machen, dass hohe Importe aus dem Süden kompatibel sind mit einem hohen Beschäftigungsniveau im Norden.

\section{Schaffung von Wachstum im Süden durch Währungspolitik}

Das andere Verfahren ist die Währungspolitik: Der Wechselkurs zwischen der Währung des Südens - nennen wir sie Rupie - und der Währung des Nordens nennen wir sie Dollar - sollte Exporte aus dem Süden in den Norden begünstigen. Hieraus sollte ein Exportüberschuss des Südens gegenüber dem Norden resultieren, dem natürlich in gleicher Höhe ein Importüberschuss des Nordens gegenüber dem Süden entspricht. Der Norden sollte somit eine unterbewertete Rupie tolerieren, so wie er in der Vergangenheit einen unterbewerteten Renminbi toleriert und damit die Exportüberschüsse Chinas ermöglicht hat.

Spricht man von einem künstlich niedrig gehaltenen Kurs der Rupie, so denkt man primär an einen Zustand, in dem die Zentralbank des Südens den Rupienkurs ausgedrückt in Dollar auf einem Niveau festlegt, welches niedriger liegt als der freie Gleichgewichtskurs, der sich ohne Zentralbankintervention bilden würde. Sie könnte dies, indem sie im großen Stil Dollar aufkauft und so große und ständig wachsende Devisenreserven anhäuft. Dieses Verfahren war das der chinesischen Zentralbank. Es kann allerdings nur gelingen, wenn der Norden eine solche Strategie nicht durch eine Gegenstrategie durchkreuzt. Man denkt in diesem Fall auch daran, dass hierfür auch Kapitalverkehrskontrollen erforderlich werden, um eine massive Spekulation auf eine Rupien-Aufwertung zu verhindern. 
Dies ist allerdings eine Betrachtungsweise, die die gesamtwirtschaftlichen Effekte einer Politik der Exportförderung für den Süden nicht hinreichend berücksichtigt. Denn dem Exportüberschuss des Südens entspricht ein entsprechend großer Nachfrageentzug für die Produkte des Nordens. Zugleich ist das Kapitalangebot im Norden entsprechend größer, weil ja dem südlichen Exportüberschuss in gleichem Ausmaß ein Kapitalexport in den Norden entspricht. Konkret: Der Süden, nicht nur, aber auch die südliche Zentralbank, sitzt auf einer wachsenden Anzahl von nördlichen Finanzierungstiteln, z.B. nördlichen Staatsanleihen. Das aber bedeutet einen niedrigeren Gleichgewichtszins für den Norden, der damit den Nachfrageentzug durch vermehrte Investitionen wettmacht, die sich bei einem höheren Zins nicht rentiert hätten. Genau dieses Phänomen der Sparschwemme auf den Weltkapitalmärkten hat die amerikanische Zentralbank in den späten neunziger und den frühen Zweitausender Jahren beobachten können, womit sie den niedrigen Zentralbankzins begründet hat. China begann in großem Stil US-amerikanische Staatsanleihen zu kaufen.

Nun kann es sein, dass der Nachfrage-kompensierende, erforderliche Gleichgewichtszins aufgrund der zusätzlichen Kapitalimporte aus dem Süden negativ wird. Will man im Norden Inflation vermeiden, und will man damit uno actu negative Realzinsens vermeiden, dann muss die Wachstumsförderung für den Süden begleitet werden durch vermehrte Staatsverschuldung im Norden. Die gewollte Verschiebung der Nachfrage von nördlichen Produkten zu südlichen Produkten muss durch eine erhöhte Gesamtnachfrage im Norden kompensiert werden, welche mithilfe zusätzlicher Staatsverschuldung zustande kommt. Wir befinden uns ja in diesem Fall in einer Situation, wo der Zinssatz (in der Nähe von Null) unter der Wachstumsrate liegt, sodass das Barro-Ricardo-Theorem nicht gilt - und deshalb zusätzliche Staatsverschuldung in der Tat zu vermehrter Gesamtnachfrage führt.

Die hier präsentierte Modellvorstellung eines NordSüd-Zweiländer-Modells kann in den folgenden Graphiken festgehalten werden.

Zuerst der Zusammenhang zwischen dem südlichen Exportüberschuss (als Ursache) und der Wachstumsrate des Südens (als Folge). In der Außenhandelstheorie spricht man hier von export led growth (vgl. Abb. 8.1).

Sodann eine etwas kompliziertere Abbildung (Abb.8.2), wobei $w$ den Wechselkurs Dollar/Rupie angibt, $D$ die Nachfrage-kompensierenden Staatsschulden im Norden, $X$ den Exportüberschuss im Süden und $g$ die Wachstumsrate im Süden.

Wir betrachten zwei Alternativen: Die blaue und die grüne. Bei der blauen Alternative ist der reale

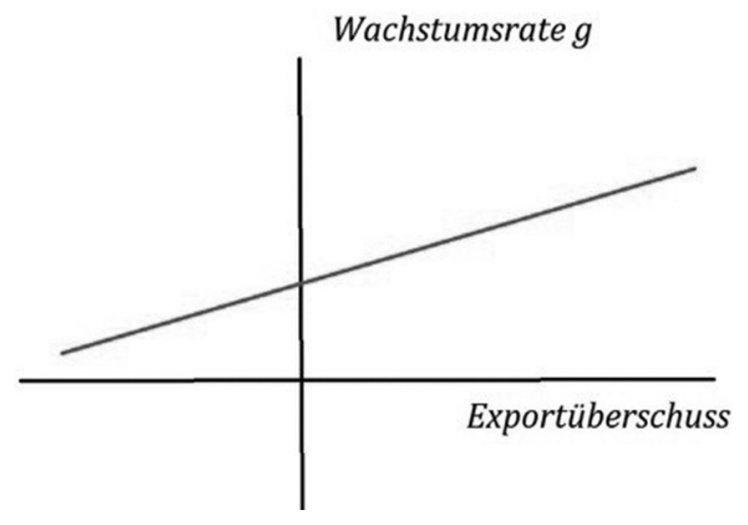

Abbildung 8.1.

Quelle: Eigene Darstellung

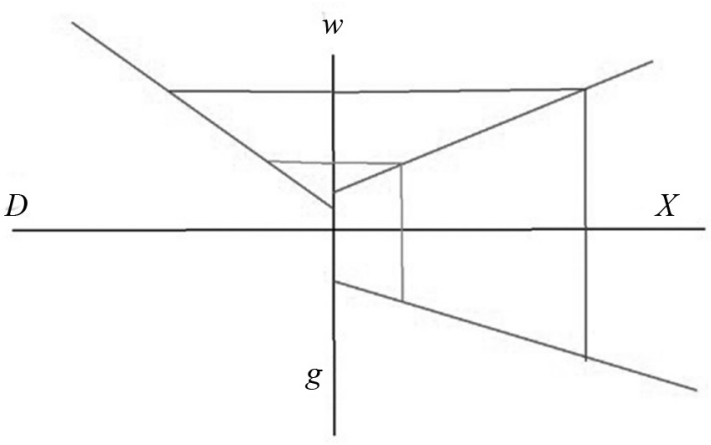

Abbildung 8.2.

Quelle: Eigene Darstellung

Wechselkurs des Dollars höher als bei der grünen. Der blauen Alternative entspricht damit ein höherer Exportüberschuss des Südens (vgl. den nordöstlichen Quadranten in Abb. 8.2). Dem höheren Exportüberschuss des Südens entspricht ein höheres Wachstum im Süden (export led growth, vgl. den südöstlichen Quadranten in Abb. 8.2). Das Ausmaß der Nachfragekompensierenden Staatsverschuldung ist in der blauen Alternative höher als in der grünen (vgl. den nordwestlichen Quadranten in Abb. 8.2).

Wir sehen daran, dass ein höheres Wachstum im Süden einher geht mit einer höheren Staatsverschuldung im Norden. Wir haben in diesem Zwei-LänderModell drei Gleichungen, die Wirkungszusammenhänge widerspiegeln; aber wir haben die vier Unbekannten $g$, $X, w, D$. Eine dieser Unbekannten muss damit exogen, sprich: Von der Wirtschaftspolitik, festgelegt werden. Das könnte der Wechselkurs $w$ sein. In diesem Fall müsste dann die Fiskalpolitik die Staatsschulden je nach Konjunkturlage nachjustieren, um so die Vollbeschäftigung im Norden aufrecht zu erhalten. Oder die Fiskalpolitik setzt von sich aus die Staatsverschuldung $D$ fest, sodass dann die Kapitalströme den entsprechenden gleichgewichtigen Wechselkurs bestimmen, 
der zu dem Exportüberschuss $X$ passt, der dem südlichen Kapitalexport entspricht. In dieser Darstellung sind natürlich die Dynamik und auch die Inflationsrate nicht mit dargestellt, die in einem ausgearbeiteten dynamischen Modell mit vorkommen.

\section{Wohlstandszuwachs im Norden}

Nun stellen wir aber außerdem fest, dass dieses höhere, stärker exportgetriebene Wachstum im Süden auch dem Wohlstand im Norden zugutekommt. Hierzu ist es gut, zwei hypothetische Fälle zu unterscheiden. Der der erste Fall ist der (den ich heutzutage nicht mehr für gegeben halte): Das gleichgewichtige RealZinsniveau $r$ ist größer als die Wachstumsrate des Nordens. In diesem Fall führt der Kapitalimport aus dem Süden dazu, dass der Zins im Norden sinkt, mit der Folge, dass hier der Wohlstand steigt (vgl. neoklassische Wachstumstheorie). Man rückt näher an die Golden Rule of Accumulation heran. Man beachte, dass hier - im Gegensatz zum Fall einer geschlossenen Volkswirtschaft - die zusätzliche Kapitalverfügbarkeit nicht durch zusätzliches heimisches Sparen generiert wird, sondern durch einen Importüberschuss. Das aber heißt, dass der Wohlstandsgewinn von Anfang an genossen werden kann. Anders ausgedrückt: Ein Verzicht auf eine Politik nach diesem Vorschlag entspricht einem Zustand dynamischer Ineffizienz.

Der zweite Fall ist der, bei dem schon vor einer solchen Politik der Zinssatz unter der Wachstumsrate des Nordens liegt. In diesem Fall kann der Staat ohne Belastung künftiger Generationen die Staatsschulden erhöhen, indem er zum Beispiel seine Steuern senkt. Auch dies ist wohlstandssteigernd. Eine detaillierte Darstellung dieses zweiten Falls ist enthalten in Weizsäcker 2015, insbesondere Abschnitt 7. Wie schon Diamond 1965 festgestellt hat, stellt im Fall der dynamischen Ineffizienz $(r<g)$ eine zusätzliche, aber nicht übertriebene Staatsverschuldung keine Belastung künftiger Generationen dar.

Das politisch zentrale Argument für eine höhere Staatsverschuldung im Fall einer Förderung der Importe aus dem Süden ist jedoch das der Aufrechterhaltung der Anzahl der Arbeitsplätze im Norden. Denn, ohne Zinssenkungen oder - im Null-Zins-Fall - ohne zusätzliche Staatsverschuldung würde die Substitution heimischer Produktion durch Importe die Arbeitsplätze im Norden schrumpfen lassen. Die effektive Nachfrage nach Gütern des Nordens wäre kleiner als ohne eine solche Politik der Importermutigung.

Wir stellen damit folgendes fest: Eine solche Politik der Förderung des südlichen Wachstums durch den
Norden ist für beide - den Süden und den Norden eine Wohlstandsverbesserung. Sie ist, wenn wir nur die beiden Aggregate Süden und Norden als Personen behandeln, eine Pareto-Verbesserung. Der Süden profitiert durch die zusätzliche Schaffung von Arbeitsplätzen und durch zusätzliches wirtschaftliches Wachstum. Der Norden profitiert erstens, weil die Zuwanderung vom Süden in den Norden mit einer solchen Politik geringer ist, als sie ohne eine solche Politik wäre. Er profitiert zweitens auch deshalb, weil er - ohne die Zukunft zu belasten - bei niedrigerem eigenfinanziertem Investitionsaufwand und mit niedrigeren Steuern bei gleicher Anzahl von Arbeitsplätzen mehr konsumieren kann.

Wir können die Intuition, die hinter diesem Politikvorschlag steht, auch so zusammenfassen: Das Weltsozialprodukt steigt mit dieser Politik, weil man von dem riesigen Arbeitskräftepotential, das bisher im Süden sehr ineffizient genutzt wird, besseren Gebrauch macht. Dadurch kann man nicht nur im Süden besser leben; sondern auch im Norden kann man entweder mehr konsumieren oder weniger arbeiten, also mehr Freizeit genießen.

\section{Zur politischen Ökonomie des Widerstandes gegen den Vorschlag 1: Status Quo Bias der Interessenvertretung}

Ich erwarte massiven Widerstand gegen diesen Vorschlag einer Wanderungsbremse. Bei einer stärker disaggregierten Betrachtung verschwindet seine Eigenschaft einer Pareto-Verbesserung. Dies zumal aus folgendem Grund:

Natürlich sind die Warenkörbe, die die beiden Volkswirtschaften produzieren, mit einer solchen Politik der Wachstumsförderung im Süden andere als ohne eine solche Politik. Das gilt selbstverständlich für den Süden, wobei hier möglicherweise von allem mehr produziert wird. Demgegenüber gibt es bisher im Norden hergestellte Produkte, die nunmehr im Süden hergestellt werden. Es gibt somit im Norden auch Verlierer bei einer solchen Politik. Die internationalen komparativen Vorteile verschieben sich. Gerade Deutschland mit seinem weit überdurchschnittlichen Anteil des produzierenden Gewerbes an der gesamtwirtschaftlichen Wertschöpfung müsste sich auf einen wuchtigen Strukturwandel einstellen. Denn es ist ja gerade das produzierende Gewerbe, das bei den internationalen Handelsströmen im Vordergrund steht. Ein für den deutschen Industriestandort ungünstiger Wechselkurs zwischen Dollar und Rupie wird in Deutschland auf Widerstand stoßen. Aber natürlich gilt 
Ähnliches auch für andere Nationalstaaten und auch für Europa insgesamt. Es kann allerdings durchaus sein, dass die deutsche Industrie von der vorgeschlagenen Politik neben der erschwerten Wettbewerbsfähigkeit auch große Vorteile bezieht, weil ein rascheres Wachstum im Süden die Weltnachfrage nach Investitionsgütern steigern müsste. Das aber käme gerade der deutschen Industrie wieder sehr zugute. Dennoch wird voraussichtlich eine forcierte Industrialisierung des Südens im Saldo auch eine Gewichtsverschiebung in Deutschland in Richtung auf mehr Dienstleistungen und weniger Industrie mit sich bringen.

Umgekehrt wird es im Norden Branchen geben, die überdurchschnittlichen Gewinn aus der Wachstumspolitik für den Süden ziehen. Jedoch ist es eine altbekannte Erkenntnis, dass in der politischen Interessenvertretung die Kräfte stärker sind, die den jeweiligen Status Quo verteidigen, als die Kräfte, die aus Strukturveränderungen Honig saugen können. Es gibt sozusagen eine starke endogene Loss-Aversion, die verbandsstrukturell in die Interessenvertretung eingebaut ist. Die Gewinne aus Strukturveränderungen sind meist diffus über einen großen Teil der Bevölkerung verteilt und auch schwer im Voraus zu quantifizieren. Sie sind daher schlecht in Lobbygruppen zu bündeln. Demgegenüber sind drohende Arbeitsplatzverluste sehr viel konkreter auszumachen und auf eine kleinere Anzahl von Branchen konzentriert. Der Widerstand gegen eine Politik, die solche Arbeitsplatzverluste mit sich bringt, ist sehr viel leichter zu mobilisieren als ein Kampf der Nutznießer dieser Politik (vgl. hierzu Olson 1965).

\section{Zur politischen Ökonomie des Widerstandes gegen den Vorschlag 2: Vorurteile gegen Staatsschulden}

Die Diskussion, ob Staatsschulden abgebaut oder vermehrt werden sollen, wird in der Öffentlichkeit meist ohne Verweis auf den Preis der Staatsschulden, sprich: Ohne Verweis auf den zu zahlenden Zins geführt. Dabei kommt es bei der Antwort auf diese Frage ganz entscheidend auf diesen Preis an. Dass die volkswirtschaftlich optimale Nutzung einer Ressource sich mit ihrem Gleichgewichtspreis ändert, sollte unter gut ausgebildeten Ökonomen nicht kontrovers sein. Daher sollte auch im öffentlichen Diskurs über Staatsschulden deren Preis eine zentrale Rolle spielen. Es soll an dieser Stelle keine ausführliche Darstellung der ökonomischen Theorie der Staatsverschuldung erfolgen (vgl. hierzu Holtfrerich et al. 2015). Wichtig ist hier vor allem ein Punkt: Es kommt entscheidend darauf an, ob der gleichgewichtige Zins ( $r$ ), den ein Fiskus auf Dauer auf seine Schulden zu zahlen hat, über oder unter der auf lange Sicht zu erwartenden Wachstumsrate $(g)$ seines Steuersubstrats liegt. Ist $r$ größer als $g$, (und ist $g$ größer als Null), dann ist es unter Ökonomen relativ unkontrovers, dass eine dauerhafte zusätzliche Staatsverschuldung problematisch ist, weil sie künftige Generationen belastet. Strittig ist die Politikempfehlung für den Fall, dass der Zins $r$ unter der nachhaltigen Wachstumsrate liegt. Einfache Wachstumsmodelle, aber auch kapitaltheoretische Überlegungen bringen das Ergebnis, dass in diesem Fall der Wohlstand durch vermehrte Staatsverschuldung gesteigert werden kann. Im Rahmen dieser Modelle kann man zeigen, dass im Fall $r<g$ eine vermehrte Staatsschuld bei gleichbleibenden Staatsausgaben den privaten Konsum dauerhaft steigen lässt. Es werden in diesem Fall durch die vermehrte Staatsschuld künftige Generationen nicht belastet (vgl. hierzu Weizsäcker 2015). Ist der reale Gleichgewichtszins im Fall der Prosperität gar negativ, dann gibt es zusätzliche Gründe für eine vermehrte Staatsverschuldung; diese sind verwandt mit der Keynesschen Theorie eines möglichen Mangels an effektiver Nachfrage.

Gegen diese modelltheoretischen Überlegungen für den Fall $r<g$ werden von anderen Ökonomen Einwände erhoben. So wird auf die Problematik hingewiesen, dass der Staat mit den ihm anvertrauten Mitteln einen verschwenderischen Gebrauch mache, was nur durch eine Schuldenbremse abgemildert werden könne. Es wird von ökonomischen Theoretikern auch in Zweifel gezogen, ob eine Situation $r<g$ eine dauerhafte Situation sei. Schließlich wird in Zweifel gezogen, ob die volkswirtschaftliche Rendite zusätzlicher privater Investitionen durch den Zinssatz wiedergegeben wird, den der Fiskus auf seine Schulden bezahlt.

An dieser Stelle will ich diese Debatte nicht weiterführen (Weizsäcker 2013). Ich erwähne sie nur, weil nicht zu erwarten ist, dass Ökonomen in dieser Sache mit einer Stimme sprechen werden. Umso wahrscheinlicher ist es, dass das Thema Staatsschulden Spielball von jeweiligen Partialinteressen wird. Die in Deutschland in der Bevölkerung heute vorherrschende Abneigung gegen Staatsschulden kann damit zusätzlich genutzt werden, um den heutigen Status Quo gegen Vorschläge wie den hier entwickelten zu verteidigen.

Es bedarf daher wohl erst einer großen Krise, ehe man sich neuen Gedanken zuwendet. Dies sei meinen nun folgenden Abschluss-Bemerkungen vorausgeschickt. 


\section{Fazit: Global-Soziale Marktwirtschaft}

Auslöser meiner Überlegungen ist die akute Flüchtlingskrise. Es spricht manches dafür, dass sich diese Krise nicht einfach mit einigen administrativen Maßnahmen der Zuwanderungsbeschränkung in kurzer Frist in Luft auflösen wird. Vermutlich ist sie Symptom für eine neue Welle der weltweiten Angleichung von Lebensbedingungen, die sich unter anderem eben in großen Wanderungen niederschlägt. Es ist dann im Interesse des Nordens diesen Angleichungsprozess zu beschleunigen, um so den Anreiz des Wanderns aus dem Süden in den Norden zu dämpfen. Eine zentrale Komponente des nördlichen Erfolgsmodells, die Soziale Marktwirtschaft, sollte daher möglichst rasch globalisiert werden. Diesem Ziel einer möglichst zügigen Verwirklichung einer Global-Sozialen Marktwirtschaft dient am besten ein Kulturwandel im Süden in Richtung auf das

\section{Literaturverzeichnis}

[1] Cairncross, Frances (1977), The Death of Distance: How the Communications Revolution is Changing Our Lives, Cambridge.

[2] Deaton, Angus (2013), The Great Escape: Health, Wealth and the Origins of Inequality, Princeton.

[3] De Tocqueville, Alexis (1835/1840), De la Démocratie en Amérique, Paris.

[4] De Tocqueville, Alexis (1856), L'Ancien Régime et la Révolution, Paris.

[5] Diamond, Peter Arthur (1965), National Debt in a Neoclassical Growth Model, American Economic Review, Vol. 55, S. 1126-1150.

[6] Goethe, Johann Wolfgang (1808), Faust - eine Tragödie, Tübingen.

[7] Hayek, Friedrich A. von (1972), Die Theorie komplexer Phänomene, Tübingen.

[8] Holtfrerich, Carl-Ludwig, Lars P. Feld und Werner Heun et al. (2015): Staatsschulden: Ursachen, Wirkungen und Grenzen, Berlin.

[9] Kissinger, Henry (2011), On China, New York.

[10] List, Friedrich (1841), Das Nationale System der Politischen Ökonomie, Stuttgart u.a.

[11] Luhmann, Niklas (1968), Die Knappheit der Zeit und die Vordringlichkeit des Befristeten, die Verwaltung, Bd. 1, S. 3-30. das nördliche Erfolgsmodell. Dieser Kulturwandel kann vor allem angestoßen werden durch Exporterfolge des Südens in den Norden. Hierzu eignet sich eine Außenwirtschaftspolitik des Nordens, die einen Exportüberschuss des Südens hervorbringt und auf diese Weise im Süden möglichst viele produktive Arbeitsplätze schafft.

Der Norden würde von einer solchen Politik nicht nur deshalb gewinnen, weil auf diese Weise der SüdNord-Wanderungsdruck gemildert wird. Darüber hinaus kann der Norden auch durch eine verbesserte internationale Arbeitsteilung gewinnen. Allerdings ist es für den Erfolg einer solchen Politik auch erforderlich, dass sich der Norden auf die damit verbundenen institutionellen Veränderungen einlässt. Insbesondere muss akzeptiert werden, dass sich die komparativen Vorteile in der Güterproduktion verschieben und dass auch Institutionen wie zum Beispiel die staatliche Schuldenbremse modifiziert, grundlegend geändert oder ganz abgeschafft werden.
[12] North, Douglass C., John Joseph Wallis, Barry R. Weingast (2009), Violence and Social Orders - A Conceptual Framework for Interpreting Recorded Human History, Cambridge.

[13] Olson, Mancur (1965), The Logic of Collective Action: Public Goods and the Theory of Groups, Cambridge.

[14] Polanyi, Karl (1944), The Great Transformation, Boston.

[15] Röpke, Wilhelm (1958), Jenseits von Angebot und Nachfrage, Erlenbach-Zürich.

[16] Weizsäcker, Carl Christian von (2013), Debatte über Staatsschulden per e-mail 11 November-1 Dezember 2013, MPI Gemeinschaftsgüter.

[17] Weizsäcker, Carl Christian von (2015), Kapitalismus in der Krise? Der negative natürliche Zins und seine Folgen für die Politik, Perspektiven der Wirtschaftspolitik, Vol. 16, S. 189-212.

[18] Weltbank (2016), International Bank für Reconstruction and Development and International Monetary Fund, Development Goals in an Era of Demographic Change, Global Monitoring Report 2015/2016. 